\title{
Valoración clínico radiológica a los 3 y 5 años de la función en prótesis fijas implantosoportadas (Sistema Branemark)
}

\author{
DONADO AZCARATE A * \\ PERIS GARCIA-PATRON $\mathbf{R}^{\mathrm{a}} \mathbf{M}$ ** \\ LOPEZ-QUILES MARTINEZ J *** \\ SADA GARCIA-LOMAS JM ****
}

\begin{abstract}
Donado Azcárate A, Peris García-Patrón Rª M, López-Quiles Martínez J, Sada García-Lomas JM. Valor clínico radiológica a los 3 y 5 años de la función en prótesis fijas implantosoportadas. (Sistema Branemark). Av Periodon Implantol. 2001; 13, 3: 23-32.
\end{abstract}

\begin{abstract}
RESUMEN
OBJETIVOS:Valorar la influencia que tiene la función protésica sobre el tejido óseo de soporte del implante.

MATERIAL Y METODO: Se estudiaron 51 prótesis fijas sobre 139 implantes Branemark colocados en el Máster de Implantes de la Facultad de Odontología de Madrid. Se compararon a los 3 y 5 años todos aquellos parámetros encargados de valorar la función de la prótesis con el grado de pérdida y calidad ósea en torno a los implantes que los soportan.

RESULTADOS: Si bien algunos parámetros prostodóncicos resultan intrascendentes otros como la estabilidad de la prótesis o la longitud del cantilever parecen tener una influencia directa sobre el estado del hueso soporte, de modo que en aquellos casos de falta de estabilidad o con extremos libres demasiado largos, la calidad y cantidad ósea va disminuyendo con el tiempo de función de la prótesis.

CONCLUSIONES: Es difícil valorar la influencia de la función protésica sobre los tejidos de soporte del implante, ya que la muestra no es grande y la cantidad de variables es muy numerosa (tipo de antagonista, material, tramo edéntulo, $\mathrm{n}^{\circ}$ de implantes,etc.). Sí parece claro que la estabilidad de la misma y la longitud del cantilever son dos factores fundamentales en el pronóstico a largo plazo del tratamiento.
\end{abstract}

\section{PALABRAS CLAVE}

Prótesis implantosoportadas; reabsorción ósea; calidad ósea.

\section{INTRODUCCIÓN}

Para valorar el éxito de un tratamiento implantológico hay que estudiar una serie de parámetros que analizan tanto la función de anclaje del implante como la estabilidad de la prótesis. Dentro de los parámetros puramente implantológicos deberemos distinguir entre aquellos dependientes del tejido

* Odontólogo. Prof. Ayudante del Departamento de Medicina y Cirugia Bucal. Facultad de Odontología. Universidad Complutense de Madrid.

** Odontólogo. Prof. Asociado del Departamento de Medicina y Cirugia Bucal. Facultad de Odontología. Universidad Complutense de Madrid.

*** Médico Estomatólogo. Prof. Asociado del Departamento de Medicina y Cirugia Bucal. Facultad de Odontología. Universidad Complutense de Madrid.

**** Cirujano Maxilofacial. M.Estomatólogo. Prof. Titular del Departamento de Medicina y Cirugia Bucal. Facultad de Odontología. Universidad Complutense de Madrid. 
óseo y los dependientes de los tejidos blandos que rodean al implante. En cuánto a los parámetros prostodóncicos habra que evaluar tanto la función como la estética, si bien este último aspecto es de muy dificil valoración pues está sujeto a las opiniones de profesional y paciente y a las expectativas iniciales de éste último. En cualquier caso es evidente que resulta imprescindible un seguimiento tanto de los parámetros puramente implantológicos como de los prostodócicos para comprobar el éxito de un tratamiento y que dichos parámetros deben permanecer estables a lo largo del tiempo para poder garantizar un éxito a largo plazo. En el presente trabajo pretendemos conocer la relación existente entre los parámetros encargados de medir la función de anclaje de la fijación (pérdida ósea y calidad ósea periimplantaria) y aquellos de los que depende el buen funcionamiento y estabilidad de la prótesis de modo que podamos conocer la posible influencia de unos sobre otros.

\section{OBJETIVOS:}

1) Comprobar la estabilidad de las prótesis implantosoportadas fijas tanto completas como parciales con más de 3 años de función en virtud de:

a) Cantidad y calidad ósea periimplantaria

b) Región tratada

c) Tipo de prótesis

\section{MATERIAL HUMANO}

El estudio clínico que se desarrolla en este trabajo ha sido realizado sobre 34 pacientes parcial o totalmente desdentados tratados por miembros del equipo de implantología con 139 implantes osteointegrados tipo Bränemark y que han sido posteriormente portadores de prótesis fijas.

\section{MÉTODO}

\section{EXPLORACIÓN RADIOLÓGICA:}

1. Realización de radiografías periapicales de todas las fijaciones mediante el sistema Porta-Rinn $(1,2,3,4)$. Para ello se coloca la película de forma ortorradial a la fijación respectiva, con sus bordes paralelos al eje longitudinal de la misma. La zona de imagen ha de extenderse por lo menos $5 \mathrm{~mm}$. dentro del hueso a ambos lados del implante hacia las fijaciones vecinas. Las estrías del implante deben aparecer nítidamente en la radiografía de tal forma que pueda examinarse la unión con el tejido óseo en el fondo de rosca. Es imprescindible que la placa se mantenga plana en el porta-film.

Mediante las radiografías periapicales realizadas durante la fase clínica del estudio pudo comprobarse la pérdida ósea marginal ocurrida durante el tiempo que llevó la prótesis en funcionamiento al establecerse una comparación con las tomadas después de la colocación de los implantes tras la fase de reposo. La realización de las radiografías fue llevada a cabo por el mismo investigador, de tal forma que existieran las menores diferencias posibles en el conjunto de todo el estudio radiográfico.

Cada una de las mediciones fue realizada por 3 investigadores diferentes de forma que se obtuvo la media de todas. Si una medición discrepaba en $0,4 \mathrm{~mm}$. o más no se tenía en cuenta y se realizaba la media con los otros dos valores (Hollender y Rockler) (5).

El análisis de los parámetros mencionados se estableció según la siguiente metodología:

\section{Pérdida ósea marginal en torno a las fijaciones:}

Se midió en la radiografía periapical la distancia tanto en mesial como en distal desde un punto de referencia tomado en la fijación (zona de unión pilarimplante) hasta la zona de contacto entre el titanio y el tejido óseo $(6,7)$. No se tuvo en cuenta aquellos puntos en los que se encontraba en contacto el hueso con el pilar, ya que se considera que en dicha zona no existe osteointegración. Mediante la fórmula (1/2 (hl +h2)) obtenemos la media de dicha pérdida en torno a la fijación, siendo hl la pérdida producida en mesial y h2 la producida en distal a la fijación (8). Como referencia interna se utilizó la medida de $0,6 \mathrm{~mm}$. entre dos espiras del implante.

\section{Pérdida ósea entre fijaciones:}

Se midió la longitud de una línea imaginaria perpendicular en su mitad a la línea de unión entre dos puntos de referencia prefijados en los implantes y que llegue hasta el nivel óseo. Este punto de referencia es la unión entre la fijación y el pilar. En las fijaciones distales hubo que tomar otro punto de referencia para trazar dicha línea (perpendicular que delimite el borde posterior de la prótesis) $(6,7)$.

\section{Calidad ósea periimplantaria:}

Se midió la calidad del tejido óseo de soporte que rodea al implante, según la siguiente tabla: nivel l=hueso trabecular en torno a la fijación, nivel 
2=hueso radiopaco marginal con hueso trabeculado en zonas más profundas, nivel 3 =hueso compacto en todo el entorno del implante $(6,7)$.

\section{EXPLORACIÓN CLÍNICA (FIGS. 1 Y 2):}

Se verifica la integridad de la prótesis comprobando la ausencia de fracturas de cualquiera de las estructuras integrantes, tanto funcionales como meramente estéticas.

Para la realización del estudio oclusal se anotó la presencia de facetas de desgaste (6) y además se comprobó la existencia de contactos prematuros en relación céntrica y de interferencias en deslizamientos laterales.

\section{Comprobación de la estabilidad de la prótesis:}

Mediante palpación y percusión con una vara de metal sobre las superficies oclusales de la prótesis inmediatamente por encima de la fijación individual (9).Gracias a la percusión con una vara de metal sobre las superficies oclusales podemos distinguir la diferencia de resonancia entre fijaciones estables y móviles (6).

Mediante sobrecarga en los extremos distales.Cuando existe una pérdida aunque sea mínima de retención entre la prótesis y una o más fijaciones, al realizar una sobrecarga en las porciones distales de la misma, se produce un sonido de tintineo característico $(6,10)$.

Medición de la longitud de la extensión protésica con una regla milimetrada desde el extremo distal de la última pieza hasta el punto de contacto distal de la última fijación con la prótesis(11). Con ello establecimos una correlación entre dicha longitud y el grado de pérdida ósea marginal de las fijaciones.

\section{ESTUDIO ESTADÍSTICO (ANÁLISIS DE RESULTADOS)}

El estudio estadístico fue realizado en el Centro de Proceso de Datos de la UCM en el seno del área de Apoyo a la Investigación. El Software estadístico utilizado ha sido el BMDP ( Dixon WJ, 1993. BMDP Statistical Software Manual. University of California Press).

\section{RESULTADOS}

\section{ANÁLISIS DESCRIPTIVO DE LOS PARÁMETROS ESTUDIADOS}

$\square$ La localización de las prótesis implantosoportadas fue la siguiente: Un $55,9 \%$ en maxilar inferior y un
$44,1 \%$ en maxilar superior (m 1,4412 sd 0,50399).

- Tipo de prótesis, el $50 \%$ son completas y el $50 \%$ en extremos libres (m 1,5000 sd 0,50752).

Antagonista: el 18,2\% ocluyen con prótesis removible de acrílico, el $24,2 \%$ con prótesis implantosoportadas y el $57,6 \%$ con dientes naturales ( $m 2,4412$ sd 0,82356).

$\square$ Integridad de la prótesis: un 58,8\% tenían integridad frente a un $41,2 \%$ que no (m 1,5882 sd 0,49955).

Estabilidad de la prótesis: un $79,4 \%$ eran estables, mientras que un $20,6 \%$ no (m 1,7941 sd 0,41043 ).

- Facetas de desgaste: existían facetas en un $41,2 \%$, no había facetas en un 58,8\% (m 1,4118 sd 0,49955).

\begin{tabular}{|l|c|c|c|}
\hline & Integridad & Estabilidad & Facetas \\
\hline SI & $58,8 \%$ & $79,4 \%$ & $41,2 \%$ \\
\hline NO & $41,2 \%$ & $20,6 \%$ & $58,8 \%$ \\
\hline
\end{tabular}

Relación entre los parámetros referentes a las prótesis implantosoportadas estudiadas:

El número de prótesis implantosoportadas estudiadas fue de 34, y los parámetros analizados fueron: la localización de las mismas, el tipo de diseño de la prótesis, el antagonista, el número de implantes que la soportaba, la integridad y estabilidad, así como la existencia de facetas de desgaste. Si bien pocas correlaciones entre estos parámetros han resultado ser estadísticamente significativos ya que el número de casos analizados no es alto, algunas de ellas son interesantes desde el punto de vista clínico y esbozan potenciales líneas de investigación especializadas en aspectos puramente protésicos, y por lo tanto serán presentadas a continuación:

Relación entre el tipo de prótesis e integridad de la misma:

Aunque se observa una mayor proporción de prótesis íntegras en las de extremo libre sobre las completas, no resulta significativa. Sobre 17 prótesis completas y 17 en extremo libre, el $26,5 \%$ de las primeras no tienen integridad frente a un $23,5 \%$ que sí, y el $14,7 \%$ de los extremos tampoco la tienen mientras que el 35,3\% sí. Es decir, hay integridad en un $70,6 \%$ de las prótesis en extremo libre, cosa que sólo ocurre en un 47,1\% de las completas.

\begin{tabular}{|l|c|c|c|}
\hline Tipo & Integridad no & Integridad sí & Total \\
\hline Completa & $26,5 \%$ & $23,5 \%$ & $50,0 \%$ \\
\hline Extremo & $14,7 \%$ & $35,3 \%$ & $50,0 \%$ \\
\hline Total & $41,2 \%$ & $58,8 \%$ & $100,0 \%$ \\
\hline
\end{tabular}




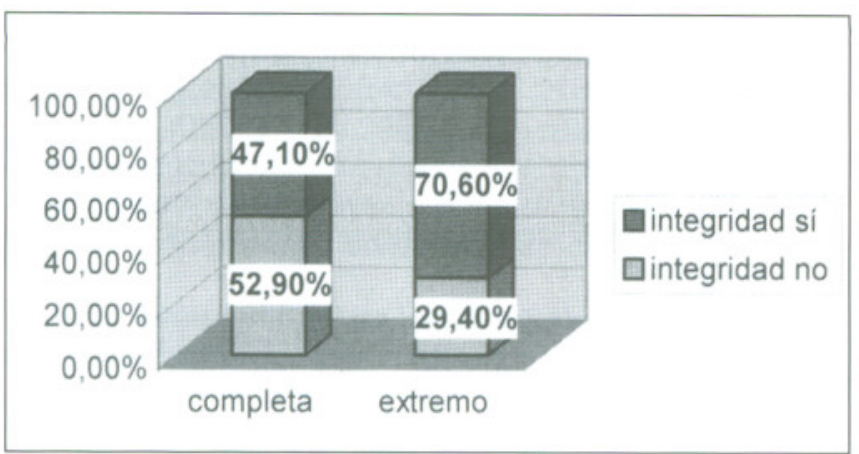

Relación entre la localización maxilar de la prótesis y la estabilidad de la misma:

En este caso sí se obtienen resultados estadísticamente significativos $(p=0,0083)$, de forma que en nuestra muestra resultaron ser claramente más estables aquellas prótesis localizadas en el maxilar superior $(100 \%)$ que las situadas en la mandíbula $(63,2 \%)$.

\begin{tabular}{|l|c|c|c|}
\hline Localización & Estabilidad no & Estabilidad sí & Total \\
\hline Inferior & $36,8 \%$ & $63,2 \%$ & $100,0 \%$ \\
\hline Superior & $0,0 \%$ & $100,0 \%$ & $100,0 \%$ \\
\hline Total & $20,6 \%$ & $79,4 \%$ & $100,0 \%$ \\
\hline
\end{tabular}

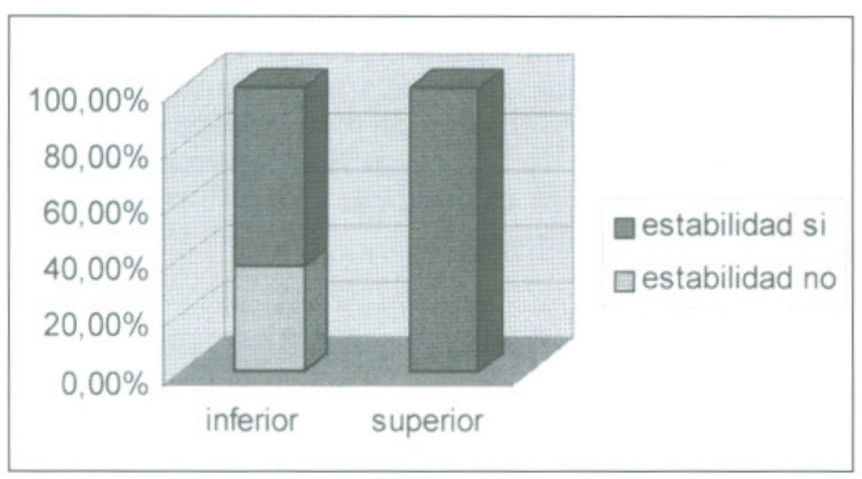

Relación entre el tipo de prótesis implantosoportada y su estabilidad:

No es significativa aunque nos da unos resultados ligeramente más favorables para las prótesis completas ( un $88,2 \%$ de estabilidad ) que para las de extremos libres ( un $70,6 \%$ de estabilidad ).

Relación entre el tipo de prótesis implantosoportada y la existencia de facetas de desgaste:

En este caso sí obtenemos un resultado estadísticamente significativo, apareciendo las prótesis comple- tas como las que presentan mayor número de facetas de desgaste (en un $58,8 \%$ de las mismas), mientras que en el caso de las prótesis en extremos libres existen en el $23,5 \%(p=0,0365)$.

\begin{tabular}{|l|c|c|c|}
\hline Tipo de prot. & Facetas no & Facetas sí & Total \\
\hline Completas & $41,2 \%$ & $58,8 \%$ & $100,0 \%$ \\
\hline Extremos & $76,5 \%$ & $23,5 \%$ & $100,0 \%$ \\
\hline Total & $58,8 \%$ & $41,2 \%$ & $100,0 \%$ \\
\hline
\end{tabular}

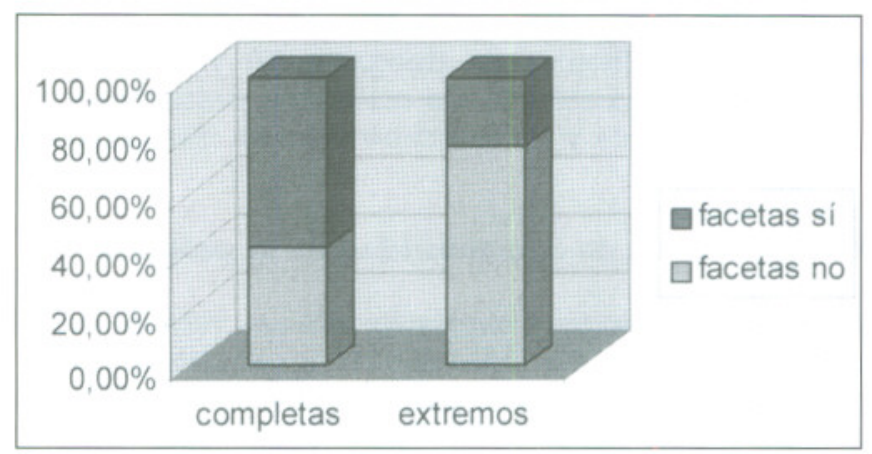

Relación entre la localización maxilar de la prótesis y el grado de reabsorción ósea sufrida por los implantes a los 3 y a los 5 años:

En el primer control radiográfico realizado a los 3 años, se comprueba, si bien no podemos decir que sea significativo, una mayor reabsorción ósea en torno a los implantes que soportan las prótesis localizadas en el maxilar superior.

La reabsorción media que se produce en las prótesis inferiores es de $0,646 \mathrm{~mm}$. y en las superiores de $0,936 \mathrm{~mm}$.

La misma circunstancia ocurre en los controles radiográficos efectuados a los 5 años:

La pérdida ósea media en los casos inferiores es de $0,738 \mathrm{~mm}$. y en los superiores de $0,958 \mathrm{~mm}$.

En cambio, las mediciones realizadas en los espacios entre los implantes tanto a los 3 como a los 5 años, señalan lo contrario:

La reabsorción media entre implantes inferiores es de $0,521 \mathrm{~mm}$. a los 3 años y de $0,750 \mathrm{~mm}$. a los 5 años, mientras que en los superiores es de 0,353 y 0,475 $\mathrm{mm}$. respectivamente.

Relación entre la calidad ósea periimplantaria y la localización maxilar de las prótesis implantosoportadas: 
Si bien a los 3 años no hay diferencia en cuanto a la calidad ósea observada en torno a los implantes superiores e inferiores, sí se aprecia una mayor calidad a los 5 años en los implantes superiores, aunque no podemos afirmar que sea estadísticamente significativo.

Relación entre el tipo de prótesis y la adaptación de los pilares transmucosos a las fijaciones:

Si bien no hay diferencias significativas, hay un mayor número de casos de falta de adaptación en las prótesis en extremos libres que en las completas. No existe ninguna falta de adaptación en estas últimas, mientras que hay un $12,7 \%$ de los extremos mal adaptados.

Relación entre el tipo de prótesis y la reabsorción ósea sufrida en torno a los implantes que la soportan a los 3 y 5 años:

Pese a que los resultados no son estadísticamente significativos, se observa que a los 3 años hay un mayor grado de reabsorción en las prótesis completas frente a los extremos libres (m 0,803 y 0,745 respectivamente).

Igualmente sucede a los 5 años y de forma más acentuada, acercándose los resultados a lo estadísticamente significativo $(t=0,0997)$. La reabsorción media en las completas es de 1,723 mm. Y en los extremos de $0,392 \mathrm{~mm}$.

Si analizamos la reabsorción ósea entre las fijaciones no se aprecian diferencias para ambos tipos de prótesis a los 3 años pero sí a los 5 (m 1,883 en las completas y 0,000 en los extremos $(t=0,0339)$.

Relación entre el tipo de prótesis y el nivel de calidad ósea periimplantaria a los 3 y 5 años:

Resulta estadísticamente significativo $(p=0,0004-t-$ $/ \mathrm{p}=0,0007-M . W .-)$ que en los controles radiográficos realizados a los 3 años se compruebe en nuestra muestra una mejor calidad ósea en torno a los implantes que soportan las prótesis en extremos libres. Este hecho también se comprueba a los 5 años si bien no es significativo desde el punto de vista estadístico.

\begin{tabular}{|l|c|c|}
\hline & Completas & Extremos \\
\hline Calidad* (m+/-s.e.m.) & $1,1686+/-0,0715$ & $1,9509+/-0,1846$ \\
\hline
\end{tabular}

(*) Calidad ósea mínima $=0 /$ máxima $=3$

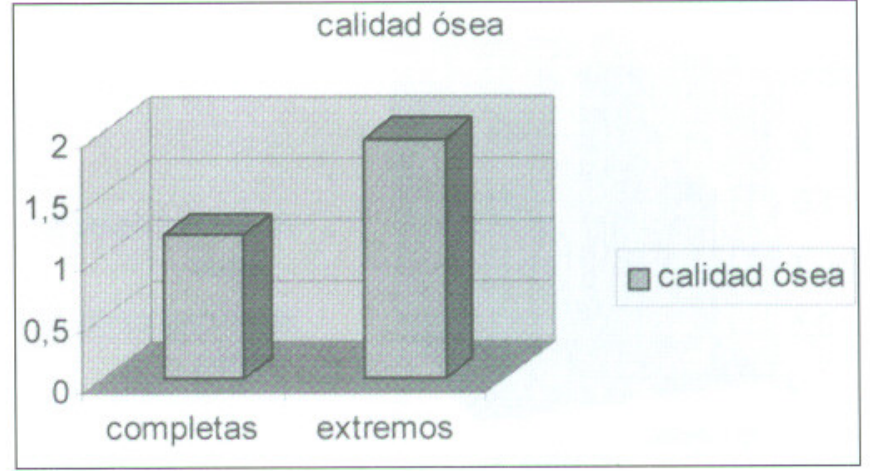

Relación entre la adaptación de la prótesis y el grado de reabsorción ósea a los 3 y 5 años:

No es posible afirmar desde un punto de vista estadístico que exista una relación entre la falta de adaptación de la prótesis y el grado de reabsorción marginal en torno a los implantes

\section{Relación entre la adaptación y la calidad ósea a los 3 y 5 años:}

Tanto a los 3 como a los 5 años se establece una relación entre estos dos parámetros de forma que la calidad ósea en torno a los implantes es peor cuando la adaptación de la prótesis es mala; ( $\mathrm{p}=0,0000$-t$/ \mathrm{p}=0,1170-\mathrm{MW}-)$ a los 3 años; $(\mathrm{p}=0,0000-\mathrm{t}-/ \mathrm{p}=0,1221-$ MW-) a los 5 años.

\begin{tabular}{|l|c|c|}
\hline & $\begin{array}{c}\text { Adaptación } \\
\text { buena }\end{array}$ & $\begin{array}{c}\text { Adaptación } \\
\text { mala }\end{array}$ \\
\hline Calidad 3 $^{\text {a }}$ (m/s.e.m.) & $1,4135+/-0,0595$ & $1,0000+/-0,0000$ \\
\hline Calidad 5 (m/s.e.m.) & $1,8966+/-0,1517$ & $1,0000+/-0,0000$ \\
\hline
\end{tabular}

Relación entre la calidad ósea y la existencia de facetas a los 3 y 5 años:

A los 3 años, se comprueba que existe una mayor calidad ósea en torno a los implantes que soportan prótesis que no tienen facetas de desgaste ( $\mathrm{t}=0,0714-\mathrm{T}-$ $/ \mathrm{t}=0,0487-\mathrm{MW}-$ ), y a los 5 años no sólo se mantiene esta tendencia sino que las diferencias son todavía mayores $(\mathrm{t}=0,0000-\mathrm{T}-\mathrm{t}=0,0004-\mathrm{MW}-)$.

\begin{tabular}{|l|c|c|}
\hline & $\begin{array}{c}\text { Facetas } \\
\text { no }\end{array}$ & $\begin{array}{c}\text { Facetas } \\
\text { sí }\end{array}$ \\
\hline Calidad 3 (m/s.e.m.) & $1,4932+/-0,0853$ & $1,2897+/-0,0741$ \\
\hline Calidad 5 (m/s.e.m.) & $2,2778+/-0,1772$ & $1,2308+/-0,1216$ \\
\hline
\end{tabular}




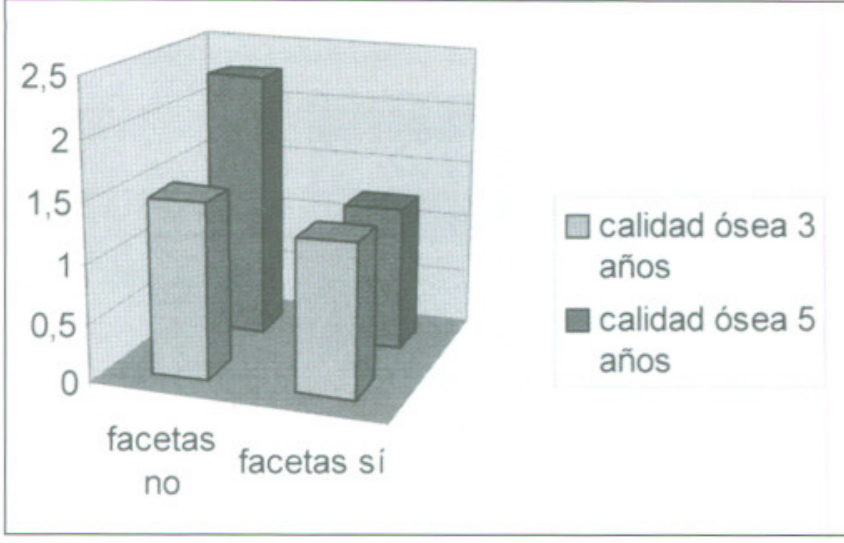

Relación entre la longitud del extremo libre y la reabsorción ósea periimplantaria:

En el control realizado a los 3 años, si bien hay una ligera tendencia a aparecer una mayor reabsorción en aquellos implantes que soportan prótesis con extremos libres más largos, desde el punto de vista estadístico la relación no resulta significativa. Sin embargo a los 5 años existe una clara correlación positiva entre mayor longitud del extremo libre y mayor grado de reabsorción marginal periimplantaria $(\mathrm{p}<0,001)$. Dicha asociación se establece a partir de la siguiente ecuación:

$$
\mathrm{Y}=0,00137+0,01405 \cdot \mathrm{X}
$$

siendo $\mathrm{Y}=$ reabsorción marginal a los 5 años. $\mathrm{X}=$ longitud del extremo libre.

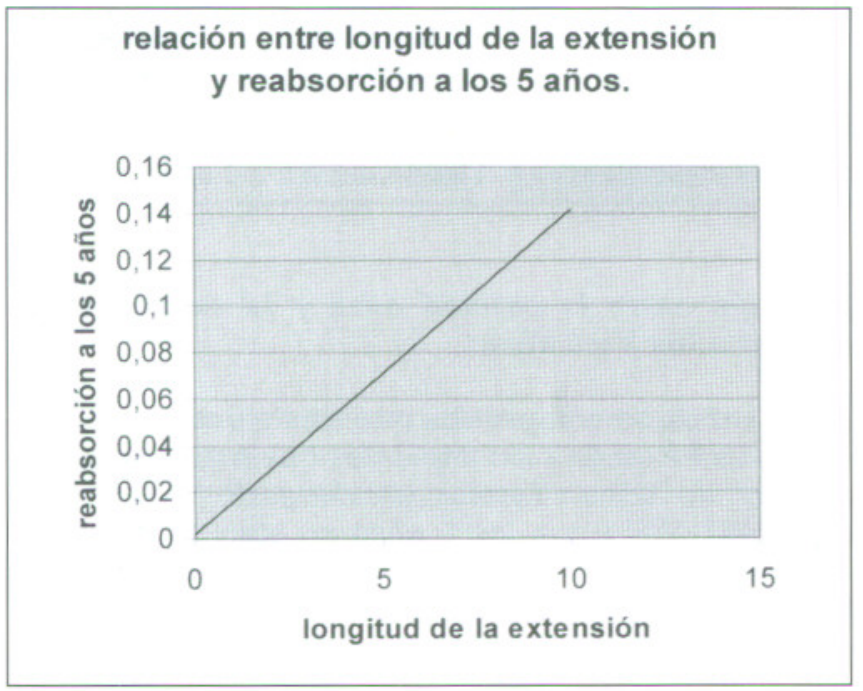

Al establecer la misma relación entre la longitud del extremo y la reabsorción sufrida en las localizaciones "entre" los implantes tal y como ha sucedido al comparar con otros parámetros, se comprueba que la asociación se mantiene y que si bien a los 3 años no es evidente, a los 5 años la pérdida ósea sufrida entre las fijaciones es mayor en aquellas prótesis con extremo libre de mayor longitud $(\mathrm{p}=0,003)$.

$$
\mathrm{Y}=-0,04721+0,01993 \cdot \mathrm{X}
$$

siendo $\mathrm{Y}=$ reabsorción entre implantes a los 5 años. $\mathrm{X}=$ longitud del extremo libre.

\section{Relación entre la longitud del extremo libre y la calidad ósea periimplantaria:}

Podemos afirmar, y el análisis estadístico así lo avala, que para nuestra muestra, tanto a los 3 años $(p<0,001)$ como los 5 años $(p<0,001)$ se observa que los implantes que soportan prótesis con extremo libre más largo presentan una peor calidad ósea en su entorno, aunque es en el segundo control cuando esta correlación es más evidente.

$$
\text { A los } 3 \text { años: } \quad \mathrm{Y}=1,9514-0,06322 \cdot \mathrm{X}
$$

siendo $\mathrm{Y}=$ calidad ósea periimplantaria a los 3 años. $\mathrm{X}=$ longitud del extremo libre.

A los 5 años: $\quad \mathrm{Y}=2,6283-0,1064 \cdot \mathrm{X}$

siendo $\mathrm{Y}=$ calidad ósea periimplantaria a los 5 años $\mathrm{X}=$ longitud del extremo libre.

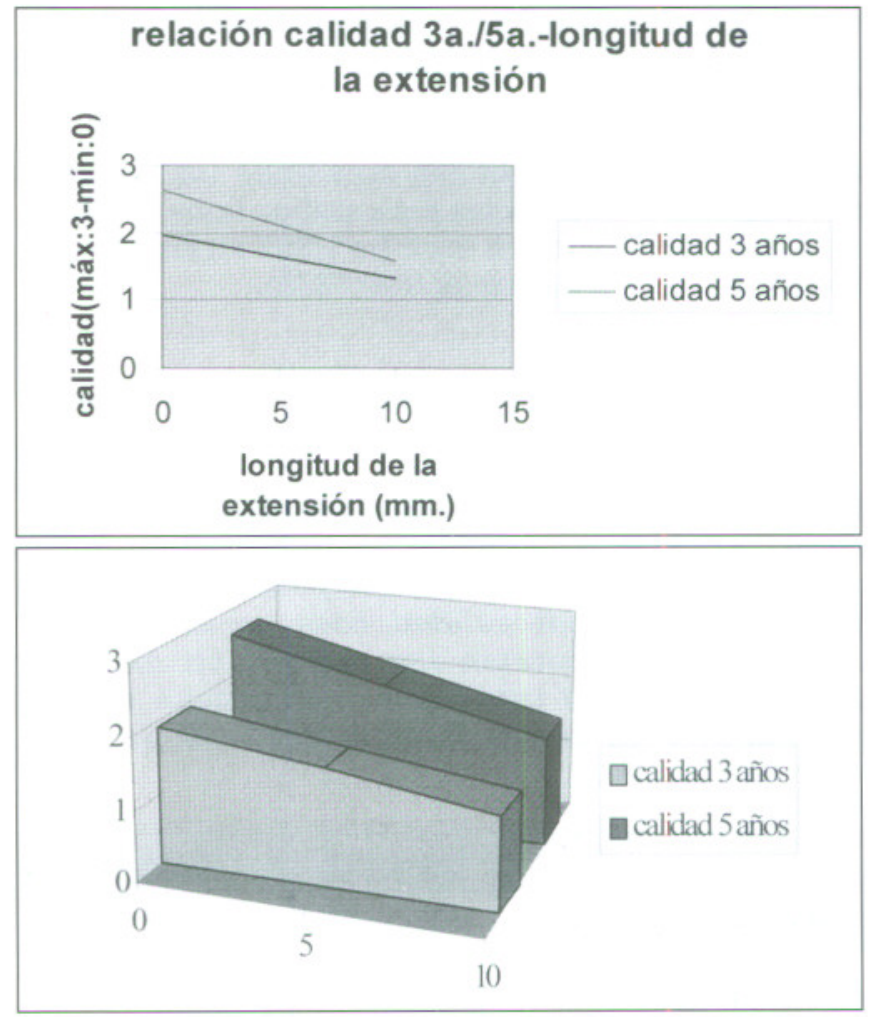




\section{Relación entre la longitud del extremo libre y la integridad de la prótesis}

Aunque se observa que precisamente las prótesis con falta de integridad son las que tienen extremos libres más largos, no podemos afirmar que exista una correlación entre ambos parámetros ya que los resultados no son estadísticamente significativos.

\section{Relación entre la longitud del extremo libre y la estabilidad de la prótesis}

Si bien se observa la misma tendencia anterior, tampoco hay evidencia estadística.

\section{DISCUSIÓN}

\section{LOCALIZACIÓN ANATÓMICA}

El grado de reabsorción ósea marginal periimplantaria, respecto a la localización maxilar de los implantes nos indica que tanto a los 3 como a los 5 años la pérdida es mayor en el maxilar superior frente al inferior. En cambio al analizar la reabsorción que tiene lugar entre las fijaciones puede observarse justamente todo lo contrario, es decir, mayor reabsorción en localizaciones mandibulares. Enfrentando entre sí ambos patrones de reabsorción los resultados parecen indicar que en las prótesis implantosoportadas superiores se da un patrón de pérdida vertical mientras que en las mandibulares el patrón es horizontal. Respecto a la calidad ósea en torno a los implantes parece superior al cabo de 5 años en los superiores aunque no resulte significativo. Adell, sin embargo, en su estudio longitudinal a 3 años, no ha encontrado diferencia alguna en cuanto a la reabsorción que se produce en maxilar superior o en mandíbula, pero sí en estudios posteriores observó mayor pérdida en los implantes más distales superiores. Nosotros en cambio, no apreciamos dicha diferencia. Ahlqvist por su parte, también encuentra una mayor reabsorción en maxilar superior más acentuada en contra de lo que podría suponerse, en los implantes más mediales frente a los laterales o posteriores (12). Los resultados obtenidos por Lindqvist avalan también estas conclusiones (13).

\section{LONGITUD DE LA EXTENSIÓN}

Otro parámetro que se ha mostrado en nuestro estudio de gran relevancia es la longitud del extremo libre ya sea en prótesis parciales en extremo o en rehabilitaciones completas. Se ha observado una clara relación en todas las prótesis estudiadas entre esta longitud y la reabsorción marginal que han sufrido los implantes, de forma que a los 5 años se demuestra que en los extremos libres de mayor longitud, sea el tipo de prótesis y localización que sea, la pérdida ósea en torno a los implantes es mayor y lo mismo sucede con el tejido óseo situado entre las fijaciones. A iguales conclusiones llega Lindqvist ya que observa una mayor pérdida en los implantes de los extremos más largos (mayor o igual a $15 \mathrm{~mm}$.) con una media de $0,95 \mathrm{~mm}$. de reabsorción, frente a los 0,61 de aquellos con extremo menor de $15 \mathrm{~mm}$..No cabe duda que éste constituye uno de los principales problemas de diseño en este tipo de prótesis ya que los estudios realizados demuestran que precisamente en las localizaciones de los extremos libres es donde el momento de las fuerzas transmitido es mayor $(14,15)$. Es por eso que algunos autores proponen soluciones para amortiguar el estrés en estas localizaciones mediante pequeños aditamentos osteointegrados que sirvan de apoyo al extremo de la prótesis (16).

También de gran trascendencia en los resultados de nuestra muestra, ha resultado ser la relación entre la mencionada longitud del extremo y la calidad ósea periimplantaria, de forma que tanto a los 3 como a los 5 años la calidad es sensiblemente peor en aquellos implantes que soportan prótesis con extremos más largos. Da igual que se trate de una prótesis parcial o de una completa. Es más, durante el segundo control se incrementa respecto al primero el número de implantes que soportando extremos largos, tienen peor calidad, con lo cual podemos suponer que el pronóstico es menos favorable en estos casos.

No hemos podido demostrar sin embargo, que exista asociación entre la longitud y la falta de integridad de la prótesis o la existencia de facetas. Aunque sí se observa que los casos suelen ser coincidentes, tal vez el número de prótesis no es suficiente para que resulte avalado por los test estadísticos. En todo caso lo que sí resulta evidente al menos en nuestra muestra es la importancia de dicha longitud en el pronóstico de los implantes en calidad y sea cual sea el tipo de prótesis que soporten.

\section{ESTABILIDAD Y OCLUSIÓN}

Respecto a la estabilidad, al analizar el estado de las prótesis superiores e inferiores resultaron ser claramente más estables las prótesis colocadas en el maxilar superior que las colocadas en mandíbula. Esta falta de estabilidad en los casos inferiores se debe exclusivamente a los extremos libres implantosoportados, ya que todos los casos de completas estudiados eran perfectamente estables. Más adelante analizaremos el porqué de esta circunstancia. También en maxilar superior las prótesis presentan menos facetas de desgaste que en mandíbula. Se observa, que en la 
muestra estudiada, hay una clara relación estadísticamente significativa entre la existencia de facetas de desgaste y una menor calidad ósea periimplantaria, de forma que en función de la localización, son como ya hemos comentado, los casos superiores, los que menor número de facetas presentan y asimismo los que mejor calidad ósea poseen en torno a los implantes.

Por otra parte es posible que pudiera establecerse una correlación entre la adaptación de la prótesis y la calidad periimplantaria, ya que los resultados estadísticos así lo insinúan si bien no son claros al respecto. Se da en nuestra muestra la circunstancia de que en aquellos casos de mala adaptación de la prótesis, la calidad ósea en torno a los implantes es peor.

\section{TIPO DE PRÓTESIS}

Si estudiamos el tipo de prótesis, comprobamos que en aquellas en extremos libres, se producen claramente mayor número de casos de falta de adaptación entre los pilares y la prótesis, de tal forma que es en éstos donde se encuentra una menor estabilidad. $\mathrm{Si}$ bien se observa que precisamente en los extremos libres hay menos casos de falta de integridad, es menor la existencia de facetas de desgaste y menor la reabsorción ósea marginal en torno a los implantes tanto a los 3 como a los 5 años, así como mejor la calidad ósea periimplantaria. Sin embargo, es en los casos concretos de prótesis inestables en extremos libres, donde se ha observado una peor calidad. Recordemos, que, como mencionamos anteriormente, la existencia de menos facetas de desgaste en la muestra general, implicaba una mejor calidad ósea. Pues bien, esto se corrobora al analizar la muestra particular de los extremos libres. Sin embargo, se ha observado que existe una correlación entre la falta de adaptación y una peor calidad ósea tanto a los 3 como a los 5 años. Es decir, podríamos concluir el análisis de los resultados en este tipo de prótesis diciendo que para nuestra muestra, la función de anclaje de los implantes y la integridad protésica es mejor en las prótesis parciales en extremos libres, pero cuando en éstas se produce una falta de adaptación, lo cual es más frecuente que en las completas, el pronóstico tanto de la prótesis como de las fijaciones empeora notablemente respecto a las anteriores. Es muy posible además que en muchos casos esta falta de adaptación conlleve finalmente la fractura de la prótesis de forma que se confirmen estudios como los de Rangert que indican un índice de fracturas muy superior en los extremos libres (17).

La falta de adaptación en este tipo de prótesis parciales, podríamos justificarla por la mayor dificultad que existe en el momento de la realización de una prótesis en un extremo libre al realizar cualquiera de los pasos necesarios ( colocación de postes de impresión, verificación de su adaptación y comprobación del ajuste pasivo de la estructura ). Pero no sólo condicionantes protésicos parecen influir en la falta de adaptación y estabilidad de la prótesis. Se observa en nuestra muestra que es frecuente la colocación poco favorable de algunos implantes en las localizaciones más distales, bien por estar demasiado próximos entre sí o lo que es más frecuente por una orientación demasiado distalizada de los mismos. Este hecho sólo se observa en los extremos libres y no en las rehabilitaciones completas, ya que en los primeros el acceso al campo operatorio es más comprometido, así como la orientación al realizar las perforaciones, especialmente en la zona mandibular debido a la curvatura ascendente que existe en la región del ángulo. Precisamente por esto último, pensamos que se da en nuestro estudio el mayor número de casos de falta de adaptación en aquellos de localización mandibular. Todas estas circunstancias probablemente habrán acentuado las dificultades anteriormente mencionadas para comprobar el ajuste correcto en las diferentes fases de realización de la prótesis. Sin embargo, esta complicación no ha tenido repercusión en la función de anclaje de los implantes ni en la existencia de facetas de desgaste, aunque sí ha provocado tres casos de fracturas ( dos de la estructura y uno de la porcelana ). Probablemente la mayor dificultad que existe para realizar un correcto ajuste oclusal en las rehabilitaciones completas frente a los tramos cortos, favorece la existencia de prematuridades o interferencias y fenómenos de frotamiento o apretamiento con la consiguiente aparición de facetas o fracturas, por otra parte favorecidas por el hecho de que la gran mayoría de las rehabilitaciones completas han sido confeccionadas en acrílico mientras todas las parciales estudiadas son de porcelana. En cualquier caso, pese a la menor incidencia de fracturas en las prótesis en extremos libres, la falta continua de estabilidad de las mismas en algunas casos produce en el paciente una gran sensación de fracaso del tratamiento, debido a la lógica falta de confianza en el momento de la masticación, y a la necesidad de recurrir continuamente al especialista para realizar ajustes, con lo cual se acentúa la percepción de la prótesis implantosoportada como cuerpo extraño y progresivamente aumenta su rechazo hasta el punto de preferir su retirada.

No nos detendremos a comentar en esta discusión la influencia que puede tener el tipo de antagonista de las prótesis estudiadas ya que aunque se conoce en el momento de la exploración no sabemos con exactitud el tiempo que llevan en función y por tanto resulta imposible analizar la influencia que este factor ha podido tener sobre otros parámetros estudiados, tales como reabsorción marginal o calidad ósea periim- 
plantaria. Sólo destacaremos que precisamente en aquellas prótesis implantosoportadas de nuestra muestra que poseen como antagonista otra prótesis igual o dentición natural, el número de fracturas es mayor. De los 13 casos en los que se observó fractura de alguna de las estructuras de la prótesis, sólo en uno el antagonista era una removible completa y el resto dentición natural o sobre implantes; lo mismo sucede en los 4 casos de falta de estabilidad en los que ninguno tenía como antagonista prótesis removibles. Estudios muy recientes de Lindqvist también encuentran un alta tasa de fracturas menores en prótesis sobre implantes que tienen antagonistas también implantosoportados (10).

\section{CONCLUSIONES}

Si bien la muestra es corta podemos aventurar que eixste una dependencia mútua entre implantes y prótesis y que en ella se basa el pronóstico a largo plazo del tratamiento implantológico. La orientación y situación de los implantes parece influir en numerosas ocasiones sobre el diseño y estabilidad de la prótesis y de igual manera la falta de ésta última parece provocar un deterioro en la función de anclaje de los implantes a medio y largo plazo. Parámetros tales como la estabilidad de la prótesis y la longitud del cantilever parecen al menos en nuestra muestra tener una relación directa con la calidad ósea en torno a los implantes así como con su reabsorción ósea marginal tras 5 años de función.

\section{ABSTRACT}

OBJETIVE: To value the influence of the prosthetic function on the supporting bone tissues.

MATERIAL AND METHOD: Fifty-one fixed prosthesis of 139 Branemark implants, placed at the Master of Implants of the Dentistry Faculty of Madrid, were studied. All parameters that value the function of the prosthesis with the degree of bone loss and quality around the implants that support them, were compared after three and five years.

RESULTS: Although some prosthodontic parameters reveal to be of no importance, others, such as the prosthesis' stability or the length of the cantilever seem to have a direct influence on the state of the supporting bone. In the case of absence of stability or too large free extremes, the bone quality and quantity diminishes during the implants' function.

CONCLUSIONS: It is difficult to value the influence of the posthetic function on the supporting tissues of the implant, due to the small sample and the large number of variable (... type, material, ... edentulous interval, number of implants, etc) It seems clear that the stability and the length of the cantilever are two fundamental factors in the long term prediction of the treatment.

\section{KEY WORDS}

Implantsupported prosthesis, bone resorption, bone quality.

\section{BIBLIOGRAFÍA}

1. Hollender L. Radiographic Examinatiion of Endosseous Implants in the Jaws. de: Advanced Osseointegration Surgery. Worthington P. and Bränemark PI. Ed Quintessence. 1992; 6: 80-93.

2. Hollender L \& Rockler B. Radiographic evaluation of osseointegrated implants of the jaws. Dentomaxillofac Radiol. 1980; 9: 91-5.

3. Jemt $\mathrm{T}$ et al. Fixed implant-supported prostheses in the edentulous maxilla. Clin Oral Impl Res. 1994; 5: 142-7.

4. Srtid KG. Resultados radiográficos de: Prótesis tejidointegradas: la osteointegración en la Odontología Clínica. Bränemark Zarb y Albrektsson Edit. Quintesence. 1987; 11: 187-98.

5. Adell R. Resultados a largo plazo del tratamiento. De: Bränemark PI y Albrektsson T Prótesis tejido-Integradas: La oseo-integración en la Odontología Clínica. Ed. Quintessence. (Ed. Española). 1987; 10: 175-86.

6. Lars $\mathrm{W}$ et al. Rehabilitación de la mandibula desdentada con prótesis fija histointegrada: un estudio longitudinal de 6 años. Quintessence (Ed. Española). 1988; 1: 41-50.

7. Zarb GA et al. The longitudinal clinical effectiveness of osseointegrated dentel implants: the toronto study. part II :the prosthetic results. J Prosthet Dent. 1989; 64: 53-61.

8. Adell $\mathrm{R}$ et al. A long -term follow-up study of osseointegrated implants in the treatment of totally edentulous jaws. Int J Oral Maxillofac Implants. 1990; 5: 347-59.

9. Buser $D$ et al. Tissue Integration of One Stage ITI Implants: 3-Years Results of a longitudinal study with Hollow-cylinder and Hollow-screw Implants. Int J Oral Maxillofac Implants. 1991; 6: 405-12. 


\section{AVANCES}

Volumen 13 - No 3 - Diciembre 2001

10. Lindqvist LW, Carlsson GE, Glantz PO. Rehabilitación de la mandíbula desdentada con prótesis fija histointegrada: un estudio longitudinal de seis años. Quintessence (Ed. Española). 1998; 1: 38-46.

11. Lars W et al. Bone resorption around fixtures in edentulous patients treated with mandibular fixed tissue-integrated prostheses. the journal of prosthettic dentistry. January. 1988; 1: 59-63.

12. Ahlqvist J, Borg K, Gunne J, Nilson H. Osseointegrated implants in edentulous jaws: a 2 year longitudinal study. Int J Oral Maxillofac Implants. 1990; 5: 155-63.

13. Lindqvist LW, Rockler B, Carlsson GE. Bone resoption around fixtures in edentulouspatients treated with mandibular fixed tissue-integrated prostheses. J of Pros Dentistry. 1988: 59; 59-63.
14. Lundgren D et al. Oclussal force pattern during mastication in denttitions with mandibular fixed partial dentures supported on osseointegrated implants. The Journal of Prosthetic Dentistry. 1987; 58: 197-203.

15. Rangert B et al. Forces and Moments on Bränemark Implants. Int J Oral Maxillofac Implants. 1989; 4: 241-7.

16. Lewwinstein I, Banks-Sills L, Eliasi R. Finite element analysis of a new system (IL) fos supporting an implantretained cantiliver prosthesis. Int J Oral Maxillofac Implants. 1995; 10: 335-66.

17. Rangert B et al. Bending overload and implant fracture: a retrospective clinical analysis. Int J Oral Maxillofac Implants. 1995; 10: 326-34. 